\title{
Generalized Fibonacci - Lucas sequence its Properties
}

\author{
Mamta Singh ${ }^{1}$, Yogesh Kumar Gupta ${ }^{2 *}$, Omprakash Sikhwal ${ }^{3}$ \\ ${ }^{1}$ Department of Mathematical Sciences and Computer Application, Bundelkhand University, Jhansi (U. P.), India \\ ${ }^{2}$ School of Studies in Mathematics, Vikram University Ujjain (M.P), India \\ ${ }^{3}$ Department of Mathematics, Mandsaur Institute of Technology Mandsaur (M. P), India \\ *Corresponding author E-mail: yogeshgupta.880@rediffmail.com
}

Copyright $\odot 2014$ Mamta Singh et al. This is an open access article distributed under the Creative Commons Attribution License, which permits unrestricted use, distribution, and reproduction in any medium, provided the original work is properly cited.

\begin{abstract}
Sequences have been fascinating topic for mathematicians for centuries. The Fibonacci sequences are a source of many nice and interesting identities. A similar interpretation exists for Lucas sequence. The Fibonacci number, Lucas numbers and their generalization have many interesting properties and applications to almost every field. Fibonacci sequence is defined by the recurrence formula $F_{n}=F_{n-1}+F_{n-2}, n \geq 2$ and $F_{0}=0, F_{1}=1$, where $F_{n}$ are an $n^{\text {th }}$ number of sequences. The Lucas Sequence is defined by the recurrence formula $L_{n}=L_{n-1}+L_{n-2}, n \geq 2$ and $L_{0}=2, L_{1}=1$, where $\mathrm{L}_{\mathrm{n}}$ an nth number of sequences are. In this paper, we present generalized Fibonacci-Lucas sequence that is defined by the recurrence relation $B_{n}=B_{n-1}+B_{n-2}, n \geq 2$ with $\mathrm{B}_{0}=2 \mathrm{~s}, \mathrm{~B}_{1}=\mathrm{s}$. We present some standard identities and determinant identities of generalized Fibonacci-Lucas sequences by Binet's formula and other simple methods.
\end{abstract}

Keywords: Fibonacci sequence, Lucas Sequence, Generalized Fibonacci sequence, Binet's Formula.

\section{Introduction}

The Fibonacci and Lucas sequences are well-known examples of second order recurrence sequences. The Fibonacci numbers are perhaps most famous for appearing in the rabbit breeding problem, introduced by Leonardo de Pisa in 1202 in his book called Liber Abaci. As illustrate in the tome by Koshy [5] the Fibonacci and Lucas number are arguable two of the most interesting sequence in all of mathematics. Many identities have been documented in an extensive list that appears in the work of Vajda [12], where they are proved by algebra means, even though combinatorial proof of many of these interesting identities. We introduced Generalized Fibonacci-Lucas Sequence and its Properties Fibonacci numbers, Lucas number's and their generalization have many interesting Properties and application to almost every field. The Fibonacci sequence [5] is a sequence of numbers starting with integer 0 and 1, where each next term of the sequence calculated as the sum of the previous two.

i.e., $F_{n}=F_{n-1}+F_{n-2}, n \geq 2$, and $F_{0}=0, F_{1}=1$

The similar interpretation also exists for Lucas sequence. Lucas sequence [7] is defined by the recurrence relation,

$\mathrm{L}_{\mathrm{n}}=\mathrm{L}_{\mathrm{n}-1}+\mathrm{L}_{\mathrm{n}-2,}, \mathrm{n} \geq 2$, and $\mathrm{L}_{0}=2, \mathrm{~L}_{1}=1$.

In this paper, we present various properties of the Generalized Fibonacci-Lucas

Sequence $\left\{\mathrm{B}_{\mathrm{n}}\right\}$ defined by $B_{n}=B_{n-1}+B_{n-2} n \geq 2$ and $\mathrm{B}_{0}=2 b, \mathrm{~B}_{1}=s$

The Binet's formula for Fibonacci sequence is given by

$F_{n}=\frac{\alpha^{n}-\beta^{n}}{\alpha-\beta}=\frac{1}{\sqrt{5}}\left\{\left(\frac{1+\sqrt{5}}{2}\right)^{n}-\left(\frac{1+\sqrt{5}}{2}\right)^{n}\right\}$ 
Where $\alpha=\frac{1+\sqrt{5}}{2}=$ Golden ratio $=1.618$

And $\beta=\frac{1-\sqrt{5}}{2}=$ Golden ratio $=-0.618$

Similarly, the Binet's formula for Lucas sequence is given by

$L_{n}=\alpha^{n}+\beta^{n}=\left\{\left(\frac{1+\sqrt{5}}{2}\right)^{n}-\left(\frac{1+\sqrt{5}}{2}\right)^{n}\right\}$

\section{Preliminary results generalized Fibonacci - Lucas sequence}

We need to introduce some basic results of Generalized Fibonacci-Lucas sequence

Generalized Fibonacci-Lucas sequence $\left\{B_{n}\right\}$ is defined by recurrence relation.

$B_{n}=B_{n-1}+B_{n-2}, \mathrm{n} \geq 2$

With initial conditions $\mathrm{B}_{0}=2 \mathrm{~s}$ and $\mathrm{B}_{1}=\mathrm{s}$ the associated initial Condition $\mathrm{B}_{0}$ and $\mathrm{B}_{1}$ are the sum of initial condition of generalized Fibonacci-Lucas sequence respectively.

i.e. $\mathrm{F}_{0}+\mathrm{b} \mathrm{L} \mathrm{L}_{0}=\mathrm{B}_{0}$ and $\mathrm{s}\left(\mathrm{F}_{1}+\mathrm{L}_{1}\right)=2 \mathrm{~B}_{1}$

The few terms of above sequence are $2 b, s, 2 b+s, 2 b+2 s, 4 b+3 s, 6 b+5 s$ and so on. The relation between Fibonacci sequence and Generalized Fibonacci-Lucas Sequence can be written as $\mathrm{B}_{\mathrm{n}}=\mathrm{F}_{\mathrm{n}}+b L_{n}, n \geq 0$.

The recurrence relation (1) has the characteristic equation $\mathrm{x}^{2}-\mathrm{x}+1=0$ this has two roots $\alpha=\frac{1+\sqrt{5}}{2}$ and

$\beta=\frac{1-\sqrt{5}}{2}$

Now notice a few things about $\alpha$ and $\beta$

$\alpha+\beta=1, \alpha-\beta=\sqrt{5}$ and $\alpha \beta=-1$

Using these two roots, we obtain Binet's recurrence relation

$$
\begin{aligned}
& B_{n}=\frac{\alpha^{n}-\beta^{n}}{\sqrt{5}}+b\left(\alpha^{n}+\beta^{n}\right) \\
& =\frac{1}{\sqrt{5}}\left\{\left(\frac{1+\sqrt{5}}{2}\right)^{n}-\left(\frac{1+\sqrt{5}}{2}\right)^{n}\right\}+b\left\{\left(\frac{1+\sqrt{5}}{2}\right)^{n}+\left(\frac{1-\sqrt{5}}{2}\right)^{n}\right\}
\end{aligned}
$$

\section{Generating function}

Now we state derive generating function of generalized Fibonacci-Lucas sequence

Generating from is $\sum_{n=0}^{\infty} B_{n} x^{n}=\frac{2 b+(s-2 b) x}{\left(1-x-x^{2}\right)}$

Let's apply power series to sequence $\left\{B_{n}\right\}$

Let $2 \mathrm{~b}+\mathrm{sx}+(2 \mathrm{~b}+\mathrm{s}) \mathrm{x}^{2}+\ldots \ldots \ldots \ldots \ldots . . . .=\sum_{n=0}^{\infty} B_{n} x^{n}$ Where $\mathrm{B}_{\mathrm{n}}$ is $\mathrm{n}^{\text {th }}$ term of sequence $\left\{B_{n}\right\}$

This is called generating series of Generalized Fibonacci - Lucas Sequence $\left\{B_{n}\right\}$

Now multiplying the generating series by the Polynomial

$$
\begin{aligned}
& \left(1-\mathrm{x}-\mathrm{x}^{2}\right) \sum_{n=0}^{\infty} B_{n} x^{n}=\sum_{n=0}^{\infty} B_{n} x^{n}-\sum_{n=0}^{\infty} B_{n} x^{n+1}-\sum_{n=0}^{\infty} B_{n} x^{n+2} \\
& =\left(\mathrm{B}_{0}+\mathrm{B}_{1} \mathrm{x}+\sum_{n=2}^{\infty} B_{n} x^{n}\right)-\left(\mathrm{B}_{0} \mathrm{x}+\sum_{n=2}^{\infty} B_{n-1} x^{n}\right)-\sum_{n=2}^{\infty} B_{n-2} x^{n}
\end{aligned}
$$




$$
\begin{aligned}
& =\mathrm{B}_{0}+\left(\mathrm{B}_{1}-\mathrm{B}_{0}\right) x+\sum_{n=2}^{\infty}\left(B_{n}-B_{n-1}-B_{n-2}\right) x^{n} \\
& =2 \mathrm{~b}+(\mathrm{s}-2 \mathrm{~b}) x+\sum_{n=2}^{\infty}\left(B_{n-1}+B_{n-2}-B_{n-1}-B_{n-2}\right) x^{n} \\
& =2 \mathrm{~b}+(\mathrm{s}-2 \mathrm{~b}) \mathrm{x}+\sum_{n=2}^{\infty}(0) x^{n} \\
& =2 \mathrm{~b}+(\mathrm{s}-2 \mathrm{~b}) \mathrm{x}
\end{aligned}
$$

Therefore, $\left(1-x-x^{2}\right) \sum_{n=0}^{\infty} B_{n} x^{n}=2 b+(s-2 b) x$

Hence $\sum_{n=0}^{\infty} B_{n} x^{n}=\frac{2 b+(s-2 b) x}{\left(1-x-x^{2}\right)}$

\section{Properties of generalized Fibonacci- Lucas sequence}

Despite its simple appearance the Generalized Fibonacci-Lucas sequence $\left\{B_{n}\right\}$ contains a wealth of subtle and fascinating properties [4], [6], [9], [12].

\section{Sum of First $n$ terms:}

Theorem 4.1: Let $B_{n}$ is the $n^{\text {th }}$ Fibonacci-Like number, and then Sum of the first $n$ terms of generalized FibonacciLucas sequence is

$$
\left(\mathrm{B}_{1}+\mathrm{B}_{2}+\mathrm{B}_{3}+\ldots \ldots . . . \mathrm{B}_{\mathrm{n}}\right)=\sum_{k=1}^{n} B_{k}=\mathrm{B}_{\mathrm{n}+2}-\mathrm{s}
$$

Proof: we know that the follows relation holds:

$\mathrm{B}_{1}=\mathrm{B}_{3}-\mathrm{B}_{2}$

$\mathrm{B}_{2}=\mathrm{B}_{4}-\mathrm{B}_{3}\left(\right.$ Since $\left.\mathrm{B}_{3}=\mathrm{B}_{2}+\mathrm{B}_{1}\right)$

$\mathrm{B}_{3}=\mathrm{B}_{5}-\mathrm{B}_{4}$

$\mathrm{B}_{\mathrm{n}-1}=\mathrm{B}_{\mathrm{n}+1}-\mathrm{B}_{\mathrm{n}}$

$\mathrm{B}_{\mathrm{n}}=\mathrm{B}_{\mathrm{n}+2}-\mathrm{B}_{\mathrm{n}+1}$

Term wise addition of all above equations, we obtain

$\left(\mathrm{B}_{1}+\mathrm{B}_{2}+\mathrm{B}_{3}+\ldots \ldots \ldots . \mathrm{B}_{\mathrm{n}}\right)=\mathrm{B}_{\mathrm{n}+2}-\mathrm{B}_{2}$

$=\mathrm{B}_{\mathrm{n}+2}-\mathrm{S}$

Sum of First $\mathbf{n}$ terms with even indices:

Theorem 4.2: Let $B_{n}$ be the $n^{\text {th }}$ Fibonacci-Lucas sequence, then Sum of the first $n$ terms with even indices is $\left(B_{2}+B_{4}+B_{6}+\ldots \ldots \ldots B_{2 n}\right)=\sum_{k=1}^{n} B_{2 k}=B_{2 n+1}-s$

Sum of First $\mathbf{n}$ terms with square indices:

Theorem 4.3: Let $B_{n}$ be the $n^{\text {th }}$ Fibonacci-Lucas sequence, then Sum of the square of first $n$ terms is $\left(B_{1}^{2}+B_{2}^{2}+B_{3}^{2}+\ldots \ldots . . B_{n}^{2}\right)=\sum_{k=1}^{n} B_{k}^{2}=B_{n} B_{n-2}$

Sum of First $\mathbf{n}$ terms with odd indices:

Theorem 4.4: Let $B_{n}$ be the $n^{\text {th }}$ Fibonacci-Lucas sequence, then Sum the first $n$ terms with odd indices is

$$
\left(B_{1}+B_{3}+B_{5}+B_{7}+\ldots \ldots \ldots . .+B_{2 n-1}\right)=\sum_{k=1}^{n} B_{2 k-1}=B_{2 n}-B_{2 n-2}
$$

Now we state and prove some nice identities similar to those obtained for Fibonacci and Lucas sequences [1], [2], [4], and [12] 


\section{Some Identities generalized Fibonacci- Lucas sequences}

In this section, some identities of Generalized Fibonacci-Lucas sequence are presented which can be easily derived by explicit sum formula and generating function.

\section{Explicit Sum Formula:}

Theorem 5.1: The explicit sum formula for Generalized Fibonacci-Lucas sequence is given by for positive integer n, prove that

$$
\mathrm{B}_{2 \mathrm{n}}=\sum_{m=0}^{n}\left(\begin{array}{l}
n \\
m
\end{array}\right) B_{n-m}
$$

Proof: By equation (2.1), it follows that

$\mathrm{B}_{2 \mathrm{n}}=\mathrm{B}_{2 \mathrm{n}-1}+\mathrm{B}_{2 \mathrm{n}-2}$

$=\left(B_{2 n-2}+B_{2 n-3}\right)+\left(B_{2 n-3}+B_{2 n-4}\right)$

$=\mathrm{B}_{2 \mathrm{n}-2}+2 \mathrm{~B}_{2 \mathrm{n}-3}+\mathrm{B}_{2 \mathrm{n}-4}$

$=\left(B_{2 n-3}+B_{2 n-4}\right)+2\left(B_{2 n-4}+B_{2 n-5}\right)+\left(B_{2 n-5}+B_{2 n-6}\right)$

$=\mathrm{B}_{2 \mathrm{n}-3}+3 \mathrm{~B}_{2 \mathrm{n}-4}+3 \mathrm{~B}_{2 \mathrm{n}-5}+\mathrm{B}_{2 \mathrm{n}-6}$

$=\mathrm{B}_{0}+\mathrm{nB}_{1}+\frac{n(n-1)}{2} B_{2}+\ldots \ldots \ldots \ldots \ldots . .+\frac{n(n-1)}{2} B_{n-2}+n B_{n-1}+B_{n}$

$\Rightarrow B_{2 n}=\sum_{m=0}^{n}\left(\begin{array}{l}n \\ m\end{array}\right) B_{n-m}$

Hence $\mathrm{B}_{2 \mathrm{n}}=\sum_{m=0}^{n}\left(\begin{array}{l}n \\ m\end{array}\right) B_{n-m}$

Theorem 5.2: The explicit sum formula for Generalized Fibonacci-Lucas sequence is given by for positive integer $n$, $\mathrm{B}_{\mathrm{n}}=\sum_{\mathrm{k}=0}^{\mathrm{n}}\left(\begin{array}{l}\mathrm{n} \\ \mathrm{k}\end{array}\right) \mathrm{B}_{\mathrm{n}-2 \mathrm{k}}$

Theorem 5.3: For every positive integer n, prove that

$$
B_{m+1} B_{n}-B_{m+1} B_{n+1}=(-1)^{n} B_{m+1} B_{n-m-1}, n \geq 1
$$

Proof: Let $\mathrm{n}$ be fixed and we Proved by inducting on $\mathrm{m}$.

When $\mathrm{m}=0$, then

$$
\begin{aligned}
& B_{1} B_{n}-B_{1} B_{n+1}=(-1)^{1} B_{1} B_{n-1} \\
& s B_{n}-s B_{n+1}=-s B_{n-1} \\
& s\left(B_{n}-B_{n+1}\right)=-s B_{n-1} \\
& s ~\left(-B_{n-1}\right)=-s B_{n-1} \\
& \text { - s } B_{n-1}=s(1+s) B_{n-1} \\
& \text { Which is true? } \\
& \text { When m=1, then } \\
& B_{1+1} B_{n}-B_{1+1} B_{n+1}=(-1)^{1} B_{1+1} B_{n-1-1} \\
& B_{2} B_{n}-B_{2} B_{n+1}=(-1)^{1} B_{2} B_{n-2} \\
& B_{2}\left(B_{n}-B_{n+1}\right)=(-1)^{1} B_{2} B_{n-2} \\
& (2 b+s)\left(B_{n}-B_{n+1}\right)=(-1)^{1}(2 b+s) B_{n-2} \\
& (2 b+s)\left(-B_{n-2}\right)=-(2 b+s) B_{n-2} \\
& -(2 b+s) B_{n-2}=-(2 b+s) B_{n-2} \\
& \text { Which also is true? }
\end{aligned}
$$

Now assume that identity is true for $m=k+1$, then by assumption

$\mathrm{B}_{\mathrm{k}} \mathrm{B}_{\mathrm{n}}-\mathrm{B}_{\mathrm{k}} \mathrm{B}_{\mathrm{n}+1}=(-1)^{\mathrm{n}} \mathrm{B}_{\mathrm{k}} \mathrm{B}_{\mathrm{n}-\mathrm{k}}$

$\mathrm{B}_{\mathrm{k}-1} \mathrm{~B}_{\mathrm{n}}-\mathrm{B}_{\mathrm{k}-1} \mathrm{~B}_{\mathrm{n}+1}=(-1)^{\mathrm{n}} \mathrm{B}_{\mathrm{k}-1} \mathrm{~B}_{\mathrm{n}-\mathrm{k}+1}$

Adding equation (5.4) and (5.5), we get

$\mathrm{B}_{\mathrm{k}} \mathrm{B}_{\mathrm{n}}+\mathrm{B}_{\mathrm{k}-1} \mathrm{~B}_{\mathrm{n}}-\mathrm{B}_{\mathrm{k}} \mathrm{B}_{\mathrm{k}+1}-\mathrm{B}_{\mathrm{k}-1} \mathrm{~B}_{\mathrm{n}+1}=(-1)^{\mathrm{n}} \mathrm{B}_{\mathrm{k}} \mathrm{B}_{\mathrm{n}-\mathrm{k}}+(-1)^{\mathrm{n}} \mathrm{B}_{\mathrm{n}-\mathrm{k}+1}$

$\left(\mathrm{B}_{\mathrm{k}}+\mathrm{B}_{\mathrm{k}-1}\right) \mathrm{B}_{\mathrm{n}}-\left(\mathrm{B}_{\mathrm{k}}+\mathrm{B}_{\mathrm{k}-1}\right) \mathrm{B}_{\mathrm{n}+1}=(-1)^{\mathrm{n}}\left(\mathrm{B}_{\mathrm{k}} \mathrm{B}_{\mathrm{n}-\mathrm{k}}+\mathrm{B}_{\mathrm{n}-\mathrm{k}+1}\right)$

$\mathrm{B}_{\mathrm{k}+1} \mathrm{~B}_{\mathrm{n}}-\mathrm{B}_{\mathrm{k}+1} \mathrm{~B}_{\mathrm{n}+1}=(-1)^{\mathrm{n}} \mathrm{B}_{\mathrm{k}+1} \mathrm{~B}_{\mathrm{n}-\mathrm{k}-1}$

Which is precisely our identity when $\mathrm{k}=\mathrm{m}$

Hence $B_{m+1} B_{n}-B_{m+1} B_{n+1}=(-1)^{n} B_{m+1} B_{n-m-1}, n \geq 1$ 
Theorem 5.4: For every positive integer $n$, prove that

$\mathrm{B}_{2 \mathrm{n}}=\mathrm{B}_{2 \mathrm{n}+1}-\mathrm{B}_{2 \mathrm{n}-1}$

Proof: we shall have proved this identity by induction matched over $\mathrm{n}$.

For $\mathrm{n}=0$

$\mathrm{B}_{2 \times 0}=\mathrm{B}_{2 \times 0+1}-\mathrm{B}_{2 \times 0-1}$

$\mathrm{B}_{\mathrm{o}}=\mathrm{B}_{1}-\mathrm{B}_{-1}$

$2 \mathrm{~b}=\mathrm{s}-(\mathrm{s}-2 \mathrm{~b})$

$2 \mathrm{~b}=\mathrm{s}-\mathrm{s}+2 \mathrm{~b}$

$2 b=2 b$

Which is also true for $\mathrm{n}=0$

When $\mathrm{n}=1$ than

$\mathrm{B}_{2 \times 1}=\mathrm{B}_{2 \times 1+1}-\mathrm{B}_{2 \times 1-1}$

$\mathrm{B}_{2}=\mathrm{B}_{3}-\mathrm{B}_{1}$

$(2 b+s)=2 b+2 s-s$

$2 b+s=2 b+s \quad$ which is also true for $n=1$

For $\mathrm{n}=\mathrm{k}$

$\mathrm{B}_{2 \mathrm{k}}=\mathrm{B}_{2 \mathrm{k}+1}-\mathrm{B}_{2 \mathrm{k}-1}$

For $\mathrm{n}=\mathrm{k}$ which is also true.

Now assume that identity is true for $n=1,2,3 \ldots \mathrm{k}$ and

We so that it holds:

For $\mathrm{n}=\mathrm{k}+1$, then by assumption

$\mathrm{B}_{2(\mathrm{k}+1)}=\mathrm{B}_{2(\mathrm{k}+1)+1}-\mathrm{B}_{2(\mathrm{k}+1)-1}$

$\mathrm{B}_{2 \mathrm{k}+2}=\mathrm{B}_{2 \mathrm{k}+3}-\mathrm{B}_{2 \mathrm{k}+1}$

$=\left(\mathrm{B}_{2 \mathrm{k}+2}+\mathrm{B}_{2 \mathrm{k}+1}\right)-\mathrm{B}_{2 \mathrm{k}+1}$

$=\mathrm{B}_{2 \mathrm{k}+2}+\mathrm{B}_{2 \mathrm{k}+1}-\mathrm{B}_{2 \mathrm{k}+1}$

$=\mathrm{B}_{2 \mathrm{k}+2}$

Which is also true, for $\mathrm{n}=\mathrm{k}+1$

Hence, the result is true for all.

Theorem 5.5: For every positive integer $n$, prove that

$\mathrm{sF}_{\mathrm{n}-1}=\mathrm{B}_{\mathrm{n}}-\mathrm{B}_{\mathrm{n}-2} \quad \mathrm{n} \geq 2$

Proof: we shall Prove this identity by induction over $n$, for $n=2$

$\mathrm{s} \mathrm{F}_{\mathrm{n}-2}=\mathrm{sF}_{2-1}=\mathrm{s} \mathrm{F}_{1}$

$=\mathrm{s} .1$

$=\mathrm{s}$

$=\mathrm{B}_{2}-\mathrm{B}_{0}$

Now suppose that identity hold for $\mathrm{n}=\mathrm{k}-1, \mathrm{n}=\mathrm{k}-2$

Then,

$\mathrm{sF}_{\mathrm{k}-2}=\mathrm{B}_{\mathrm{k}-1}-\mathrm{B}_{\mathrm{k}-3}$

$\mathrm{s} \mathrm{F}_{\mathrm{k}-3}=\mathrm{B}_{\mathrm{k}-2}-\mathrm{B}_{\mathrm{k}-4}$

On adding equation (5.8) \& (5.9) we get,

s $\mathrm{F}_{\mathrm{k}-2}+\mathrm{s} \mathrm{B}_{\mathrm{k}-3}=\left(\mathrm{B}_{\mathrm{k}-1}+\mathrm{B}_{\mathrm{k}-2}\right)-\left(\mathrm{B}_{\mathrm{k}-3}+\mathrm{B}_{\mathrm{k}-4}\right)$

$\mathrm{s}\left(\mathrm{F}_{\mathrm{k}-2}+\mathrm{B}_{\mathrm{k}-3}\right)=\mathrm{B}_{\mathrm{k}}-\mathrm{B}_{\mathrm{k}-2}$

s $\mathrm{F}_{\mathrm{k}-1}=\mathrm{B}_{\mathrm{k}}-\mathrm{B}_{\mathrm{k}-2}$

Which is true for $\mathrm{n}=\mathrm{k}$,

$\mathrm{s} \mathrm{F}_{\mathrm{n}-1}=\mathrm{B}_{\mathrm{n}}-\mathrm{B}_{\mathrm{n}-2} \quad \mathrm{n} \geq 2$

Theorem 5.6: For every positive integer n,

$B_{3}+B_{6}+B_{9}+\ldots \ldots+B_{3 n}=\frac{1}{2}\left[B_{3 n+2}-(2 b+s)\right]$

Proof: By using Binet's formula, we have

$$
\begin{aligned}
& B_{3}+B_{6}+B_{9}+\ldots \ldots+B_{3 n} \\
& =\frac{\alpha^{3}-\beta^{3}}{\sqrt{5}}+b\left(\alpha^{3}-\beta^{3}\right)+\frac{\alpha^{6}-\beta^{6}}{\sqrt{5}}+b\left(\alpha^{6}-\beta^{6}\right)+\ldots . .+\frac{\alpha^{3 n}-\beta^{3 n}}{\sqrt{5}}+b\left(\alpha^{3 n}-\beta^{3 n}\right) \\
& =\frac{1}{\sqrt{5}}\left[\left(\alpha^{3}+\alpha^{6}+\alpha^{9} \ldots . \alpha^{3 n}\right)-\left(\beta^{3}+\beta^{6}+\beta^{9} \ldots \ldots \beta^{3 n}\right)\right]+b\left[\left(\alpha^{3}+\alpha^{6}+\alpha^{9} \ldots . \alpha^{3 n}\right)-\left(\beta^{3}+\beta^{6}+\beta^{9} \ldots . \beta^{3 n}\right)\right]
\end{aligned}
$$




$$
\begin{aligned}
& =\frac{1}{\sqrt{5}}\left[\left(\frac{\alpha^{3 n+3}-\alpha^{3}}{\alpha^{3}-1}\right)-\left(\frac{\beta^{3 n+3}-\beta^{3}}{\beta^{3}-1}\right)\right]+b\left[\frac{\alpha^{3 n+3}-\alpha^{3}}{\alpha^{3}-1}+\frac{\beta^{3 n+3}-\beta^{3}}{\beta^{3}-1}\right] \\
& =\frac{1}{\sqrt{5}}\left[\frac{\alpha^{3 n+2}-\alpha^{2}}{2}-\left(\frac{\beta^{3 n+2}-\beta^{2}}{2}\right)\right]+b\left[\frac{\alpha^{3 n+2}-\alpha^{2}}{2}+\frac{\beta^{3 n+2}-\beta^{2}}{2}\right] \\
& =\frac{1}{2}\left[\frac{\alpha^{3 n+2}-\beta^{3 n+2}}{\sqrt{5}}+b\left(\alpha^{3 n+2}+\beta^{3 n+2}\right)\right]+b\left[\frac{\alpha^{2}+\beta^{2}}{\sqrt{5}}+b\left(\alpha^{2}+\beta^{2}\right)\right] \\
& =\frac{1}{2}\left(B_{3 n+2}-B_{2}\right) \\
& =\frac{1}{2}\left[B_{3 n+2}-(2 b+s)\right]
\end{aligned}
$$

This is completes the proof.

Theorem 5.7 : For every positive integer $B_{5}+B_{8}+B_{11}+\ldots \ldots .+B_{3 n+2}=\frac{B_{3 n+4}-(4 b+3 s)}{2}$

Proof: By using Binet's formula, we have

$$
\begin{aligned}
& =\frac{\alpha^{5}-\beta^{5}}{\sqrt{5}}+b\left(\alpha^{5}-\beta^{5}\right)+\frac{\alpha^{8}-\beta^{8}}{\sqrt{5}}+b\left(\alpha^{8}-\beta^{8}\right)+\ldots . .+\frac{\alpha^{3 n+2}-\beta^{3 n+2}}{\sqrt{5}}+b\left(\alpha^{3 n+2}-\beta^{3 n+2}\right) \\
= & \frac{1}{\sqrt{5}}\left[\left(\alpha^{5}+\alpha^{8}+\alpha^{11} \ldots . \alpha^{3 n+2}\right)-\left(\beta^{5}+\beta^{8}+\beta^{11} \ldots . \beta^{3 n+2}\right)\right]+b\left[\left(\alpha^{5}+\alpha^{8}+\alpha^{11} \ldots . \alpha^{3 n+2}\right)-\left(\beta^{5}+\beta^{8}+\beta^{11} \ldots . . \beta^{3 n+2}\right)\right] \\
= & \frac{1}{\sqrt{5}}\left[\left(\frac{\alpha^{3 n+5}-\alpha^{5}}{\alpha^{5}-1}\right)-\left(\frac{\beta^{3 n+5}-\beta^{5}}{\beta^{5}-1}\right)\right]+b\left[\frac{\alpha^{3 n+5}-\alpha^{5}}{\alpha^{5}-1}+\frac{\beta^{3 n+5}-\beta^{5}}{\beta^{5}-1}\right] \\
= & \frac{1}{\sqrt{5}}\left[\frac{\alpha^{3 n+4}-\alpha^{4}}{2}-\left(\frac{\beta^{3 n+4}-\beta^{4}}{2}\right)\right]+b\left[\frac{\alpha^{3 n+4}-\alpha^{4}}{2}+\frac{\beta^{3 n+4}-\beta^{4}}{2}\right] \\
= & \frac{1}{2}\left[\frac{\alpha^{3 n+4}-\beta^{3 n+4}}{\sqrt{5}}+b\left(\alpha^{3 n+4}+\beta^{3 n+4}\right)\right]+b\left[\frac{\alpha^{4}+\beta^{4}}{\sqrt{5}}+b\left(\alpha^{4}+\beta^{4}\right)\right] \\
= & \frac{\left(B_{3 n+4}-B_{4}\right)}{2} \\
= & \frac{\left[B_{3 n+4}-(4 b+3 s)\right]}{2}
\end{aligned}
$$

This is completes the proof.

Theorem 5.8: For positive integer $n$, prove that

$$
B_{n}^{2}=(-1)^{n+1} s B_{n}, n \geq 1
$$

This can be derived same as theorem

Theorem 5.9: For every integer $n \geq 0$, prove that

$$
B_{2 n}=F_{2 n}+b L_{2 n} n \geq 0
$$

This can be derived same as theorem

Theorem 5.10: For every integer $n \geq 0$, prove that

$$
B_{n}=F_{n}+b L_{n} \quad n \geq 0
$$

This can be derived same as theorem 


\section{Connection formulae}

In this section, connection formulae of Generalized Fibonacci-Lucas sequence, induction method are presented.

Theorem 6.1: For positive integer $n$, prove that

$2 b F_{n-1}=B_{n-1}-B_{n-2}, n \geq 3$

Proof: We shall prove this identity by induction It is easy to show that for $n=3$

$2 \mathrm{bF}_{\mathrm{n}-1}=2 \mathrm{bF}_{3-1}=2 \mathrm{bF}_{2}$

$=2 \mathrm{bF}_{2}$

$=2 \mathrm{~b} .1=2 \mathrm{~b}$

$=\mathrm{B}_{2} \cdot \mathrm{B}_{1}$

Now suppose the identity holds $\mathrm{n}=\mathrm{k}-1, \mathrm{n}=\mathrm{k}-2$. Then,

$2 \mathrm{~b} \mathrm{~F}_{\mathrm{k}-2}=\mathrm{B}_{\mathrm{k}-2}-\mathrm{B}_{\mathrm{k}-3}$

$2 \mathrm{~b} \mathrm{~F}_{\mathrm{k}-3}=\mathrm{B}_{\mathrm{k}-3}-\mathrm{B}_{\mathrm{k}-4}$

On adding equation (6.2) and (6.3), we get

i.e. $2 b F_{k-2}+2 b F_{k-3}=\left(B_{k-2}+B_{k-3}\right)-\left(B_{k-3}+B_{k-4}\right)$

$2 \mathrm{~b}\left(\mathrm{~F}_{\mathrm{k}-2}+\mathrm{F}_{\mathrm{k}-3}=\mathrm{B}_{\mathrm{k}-1}-\mathrm{B}_{\mathrm{k}-2}\right.$

$2 \mathrm{~b} \mathrm{~F}_{\mathrm{k}-1}=\mathrm{B}_{\mathrm{k}-1}-\mathrm{B}_{\mathrm{k}-2}$

Which is precisely our identity when $\mathrm{n}=\mathrm{k}$

Hence $2 b F_{n-1}=B_{n-1}-B_{n-2}, \quad n \geq 3$

Theorem 6.2: For positive integer $n$, prove that

$2 \mathrm{bL}_{\mathrm{n}-1}=\mathrm{B}_{\mathrm{n}}-\mathrm{B}_{\mathrm{n}-1}, \quad \mathrm{n} \geq 2$

Proof: We shall Prove this identity by induction over $n$. for $n=2$

$2 \mathrm{bL}_{\mathrm{n}-1}=2 \mathrm{~b} \mathrm{~L}_{2-1}=2 \mathrm{~b} \mathrm{~L}_{1}$

$=2 \mathrm{~b} .1$

$=2 \mathrm{~b}$

$=\mathrm{B}_{2}-\mathrm{B}_{1}$

Now suppose the identity holds for $\mathrm{n}=\mathrm{k}-1, \mathrm{n}=\mathrm{k}-2$. Then,

$2 \mathrm{~b} \mathrm{~L}_{\mathrm{k}-2}=\mathrm{B}_{\mathrm{k}-1}-\mathrm{B}_{\mathrm{k}-2}$

$2 \mathrm{~b} \mathrm{~L} \mathrm{~L}_{\mathrm{k}-3}=\mathrm{B}_{\mathrm{k}-2}-\mathrm{B}_{\mathrm{k}-3}$

Adding equation (6.5) and (6.6), we get

i.e. $2 b\left(L_{k-2}+L_{k-3}\right)=\left(B_{k-1}+B_{k-2}\right)-\left(B_{k-2}+B_{k-3}\right)$

$2 \mathrm{~b} \mathrm{~L}_{\mathrm{k}-1}=\mathrm{B}_{\mathrm{k}}-\mathrm{B}_{\mathrm{k}-1}$

Which is true for $\mathrm{n}=\mathrm{k}$,

Hence $2 b L_{n-1}=B_{n}-B_{n-1}, \quad n \geq 2$

Theorem 6.3: For positive integer $n$, prove that $\mathrm{s} \mathrm{L}_{\mathrm{n}-1}=\mathrm{B}_{\mathrm{n}-1}-\mathrm{F}_{\mathrm{n}-2,}, \mathrm{n} \geq 2$

Theorem 6.4: For positive integer $n$, prove that $\mathrm{s} \mathrm{L}_{\mathrm{n}-1}=\mathrm{B}_{\mathrm{n}}-\mathrm{B}_{\mathrm{n}-2, \mathrm{n}} \mathrm{n}$

Theorem 6.4: For positive integer $n$, prove that

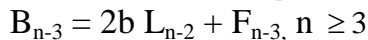

Theorem 6.5: For positive integer $n$, prove that $2 \mathrm{~b} \mathrm{~F}_{\mathrm{n}-1}=\mathrm{B}_{\mathrm{n}+1}-2 \mathrm{~B}_{\mathrm{n}-1}, \quad \mathrm{n} \geq 2$

\section{Some determinant identities}

Determinants have played a significant part in various areas in mathematics. There are different perspectives on the study of determinants. Problems on determinants of Fibonacci sequence and Lucas sequence are appeared in various issues of Fibonacci Quarterly. Many determinant identities of generalized Fibonacci sequence are discussed in [12]. In this section some determinant identities of generalized Fibonacci-Lucas sequence are derived. Entries of determinants are satisfying the recurrence relation of generalized Fibonacci-Lucas sequence and other sequences.

Theorem 7.1: Let $n$ be a positive integer. Then 


$$
\begin{aligned}
& \left|\begin{array}{ccc}
\mathrm{B}_{\mathrm{n}} & \mathrm{F}_{\mathrm{n}} & 1 \\
\mathrm{~B}_{\mathrm{n}+1} & \mathrm{~F}_{\mathrm{n}+1} & 1 \\
\mathrm{~B}_{\mathrm{n}+2} & \mathrm{~F}_{\mathrm{n}+2} & 1
\end{array}\right|=\left[F_{n} B_{n+1}-B_{n} F_{n+1}\right] \\
& \text { Proof: Let } \Delta=\left|\begin{array}{ccc}
\mathrm{B}_{\mathrm{n}} & \mathrm{F}_{\mathrm{n}} & 1 \\
\mathrm{~B}_{\mathrm{n}+1} & \mathrm{~F}_{\mathrm{n}+1} & 1 \\
\mathrm{~B}_{\mathrm{n}+2} & \mathrm{~F}_{\mathrm{n}+2} & 1
\end{array}\right|
\end{aligned}
$$

And assume $B_{n}=a, B_{n+1}=b, B_{n+2}=a+b$

$\mathrm{F}_{\mathrm{n}}=\mathrm{P}, \mathrm{F}_{\mathrm{n}+1}=\mathrm{q}, \mathrm{F}_{\mathrm{n}+2}=\mathrm{p}+\mathrm{q}$

Now substituting the value of equation (7.2) \& (7.3) in (7.1), we get

$\Delta=\left|\begin{array}{ccc}a & p & 1 \\ b & q & 1 \\ a+b & p+q & 1\end{array}\right|$

Applying $\mathrm{R}_{1} \rightarrow R_{1}-R_{2} \quad \Delta=\left|\begin{array}{ccc}a-b & p-q & 0 \\ b & q & 1 \\ a+b & p+q & 1\end{array}\right|$

Applying $\mathrm{R}_{2} \rightarrow R_{2}-R_{3} \quad \Delta=\left|\begin{array}{ccc}a-b & p-q & 0 \\ b-(a+b) & q-(p+q) & 0 \\ a+b & p+q & 1\end{array}\right|$

$\Delta=\left|\begin{array}{ccc}a-b & p-q & 0 \\ -a & -p & 0 \\ a+b & p+q & 1\end{array}\right|$

$\Delta=[p b-a q]$

Again substituting the values of the equation (7.2) and (7.3) in (7.4), we get $\Delta=\left[F_{n} B_{n+1}-B_{n} F_{n+1}\right]$

Hence $\left|\begin{array}{ccc}B_{n} & F_{n} & 1 \\ B_{n+1} & F_{n+1} & 1 \\ B_{n+2} & F_{n+2} & 1\end{array}\right|=\left[F_{n} B_{n+1}-B_{n} F_{n+1}\right]$ similarly we can derive following identities:

Theorem 7.2: For every integer $n \geq 2$, prove that $\left|\begin{array}{ccc}B_{n} & B_{n+1} & B_{n+2} \\ B_{n+2} & B_{n} & B_{n+1} \\ B_{n+1} & B_{n+2} & B_{n}\end{array}\right|=2\left(B_{n}^{3}+B_{n+1}^{3}\right)$

Theorem 7.3: For any integer $n \geq 0$, prove that $\left|\begin{array}{ccc}B_{n} & L_{n} & 1 \\ B_{n+1} & L_{n+1} & 1 \\ B_{n+2} & L_{n+2} & 1\end{array}\right|=2\left(L_{n} B_{n+1}-B_{n} L_{n+1}\right)$

Theorem 7.4: For every positive integer n, prove that $\left|\begin{array}{ccc}B_{n}+B_{n+1} & B_{n+1}+B_{n+2} & B_{n+2}+B_{n} \\ B_{n+2} & B_{n} & B_{n+1} \\ 1 & 1 & 1\end{array}\right|=0$

Theorem 7.5: For every positive integer n, prove that $\left|\begin{array}{ccc}1+B_{n} & B_{n+1} & B_{n+2} \\ B_{n} & 1+B_{n+1} & B_{n+2} \\ B_{n} & B_{n+1} & 1+B_{n+2}\end{array}\right|=1+B_{n}+B_{n+1}+B_{n+2}$

The identities from (7.1) to (7.4) can be proved similarly.

\section{Conclusion}

There are many know identities established for this paper Fibonacci and Lucas sequence. Their paper describes comparable identities of Generalized Fibonacci-Lucas sequence, Fibonacci sequence and Lucas sequence respectively. It is easy to discover new identities simply by varying the pattern of know identities and using inductive reasoning to guess new result. 


\section{References}

[1] A.F. Horadam, the Generalized Fibonacci Sequences, the American Math Monthly, 68, No 5 (1961), 455-4592.

[2] A.T. Benjamin and j. j. Quinn. Recounting Fibonacci and Lucas identities collage Math, J., No.5 (1999), $359-366$.

[3] Fuller E. Leonard, Generating function for recurrence relations, The Fibonacci Quarterly 19 (1981) 107-110.

[4] J. Z. Lee and Some Properties of Generalization of the Fibonacci sequence, The Fibonacci Quarterly, 1987, No.2, 110-117.

[5] Koshy, T. Fibonacci and Lucas number with application, Wiley, 2001.

[6] L. Carlitz, A note on Fibonacci number, The Fibonacci Quarterly, 2(1964), 15-28.

[7] L. Carlitz, Some Fibonacci and Lucas identities, The Fibonacci Quarterly, 8(1970), 61-73.

[8] Singh, B. and Sikhwal, O. Generalized Fibonacci sequence and Analytical Properties, Vikram Mathematical Journal, 26 (2006), $131-144$.

[9] Singh, B. and Sikhwal O. and Bhatnagar, S., Fibonacci-like Sequence \& its Properties, Int. Contemp Math. Science, Vol. 5, (2010) No. 18, 857-868.

[10] Singh, B., Sikhwal, O. Jain, S Coupled Fibonacci Sequences of Fifth Order and Some Properties Int. J. of Math Analysis, 4, (2010) 1247-1254

[11] Singh, B., Sikhwal, O. Fibonacci-Triple sequences and Some Fundamental Properties, Tamkang. J. of Mathematics. Sciences, 41, (2010) 325333.

[12] Sikhwal, O., Generalization of Fibonacci sequence: An Intriguing Sequence, Lap Lambert Academic Publishing GmbH \& Co. KG, Germany (2012).

[13] Vajda, S. Fibonacci and Lucas numbers and the golden section, Ellis Horwood Limited, Chi Chester, England, 1989.

[14] Vorobyou, N.N., The Fibonacci number, D.C. health company, Boston, 1963. 